\title{
COLENGTH OF DERIVATION IDEALS
}

\author{
KENNETH KRAMER
}

\begin{abstract}
In this paper, $D$ is a derivation acting on the formal power series ring $K\left[\left[t_{1}, \cdots, t_{r}\right]\right]$ over a field $K$ of characteristic $p \neq 0$. We conjecture that the colengths of the ideals $\left(D^{p^{n}} t_{1}, \cdots\right.$, $\left.D^{p^{n}} t_{r}\right)$ and $\left(D^{p^{n-1}} t_{1}, \cdots, D^{p^{n-1}} t_{r}\right)$ are congruent modulo $p^{n}$, provided they are finite. We give a proof for the case $r=1$ and any $n \geqq 1$, and for the case $n=1$ and any $r \geqq 1$.
\end{abstract}

1. Introduction. Let $A=K\left[\left[t_{1}, \cdots, t_{r}\right]\right]$ be the ring of formal power series in $r$-variables over a field $K$ of characteristic $p \neq 0$. The colength of an ideal $\mathfrak{A}$ of $A$ is the dimension of the quotient ring $A / \mathfrak{A}$ viewed as a vector space over $K$. Given $D$, a $K$-derivation of $A$, we let $\mathfrak{A}(D)$ denote the ideal $\left(D t_{1}, \cdots, D t_{r}\right)$ of $A$. Since $D^{p}$ is again a derivation, we have a descending chain of ideals:

$$
\mathfrak{A}(D) \supset \mathfrak{A}\left(D^{p}\right) \supset \mathfrak{A}\left(D^{p^{2}}\right) \supset \cdots .
$$

Conjecture. Suppose $D$ is a K-derivation of $A$. Let $i(n)$ denote the colength of the ideal $\mathfrak{A}\left(D^{p^{n}}\right)$. Then the congruence $i(n) \equiv i(n-1)\left(\right.$ modulo $\left.p^{n}\right)$ holds for each integer $n \geqq 1$ such that $i(n)<\infty$.

This paper contains a proof of the conjecture in the one-variable case and a proof of the initial congruence, $i(1) \equiv i(0)$ (modulo $p$ ), in the general case.

Suppose that $\sigma$ is a $K$-automorphism of $A$, and let 1 denote the identity automorphism of $A$. We may view the operator $L=\sigma-1$ as a "twisted derivation" in the sense that $L(x y)=x L(y)+\sigma(y) L(x)$.

Consider the analog of the above conjecture obtained by substituting $L$ for $D$. In the one-variable case, the congruences of this analog are implied by the Hasse-Arf theorem as generalized by Sen [2]. For general $A$ and $\sigma$ of finite order, the analog would follow from an affirmative answer to a question asked by Serre [4, p. 418].

The author wishes to thank John Tate for his generous advice on this paper.

Received by the editors November 30, 1972.

AMS (MOS) subject classifications (1970). Primary 13B10, 13B15, $13 \mathrm{~J} 05$.

(c) American Mathematical Society 1973 
2. The one-variable case, $A=K[[t]]$. For $\alpha \in A$, let $\alpha_{t}$ denote the formal partial derivative of $\alpha$ with respect to $t$. Thus $D \alpha=\alpha_{t} D t$ for all derivations $D$.

Lemma. Let $D$ be a K-derivation of $A$. There exists a "constant" $c \in K\left[\left[t^{p}\right]\right]$, such that $D^{s+p-1} t=c D^{s} t$, for $s \geqq 1$.

Proof. Let $D t=f$, which we may assume is not zero. As $\mathfrak{A}\left(D^{p}\right) \subset$ $\mathfrak{U}(D)$, we may write $D^{p} t=c D t$ for some $c \in A$. Now $D$ and $D^{p}$ are derivations, so

and

$$
D^{p+1} t=D\left(D^{p} t\right)=D(c f)=c D f+f D c
$$

$$
D^{p+1} t=D^{p}(D t)=D^{p} f=f_{t} D^{p} t=f_{t} c f=c D f .
$$

It follows that $f D c=0$. Hence, $c \in K\left[\left[t^{p}\right]\right]$. Now for $s \geqq 1$, we have

$$
D^{s+p-1} t=D^{s-1}\left(D^{p} t\right)=D^{s-1}(c D t)=c D^{s} t .
$$

Let $v$ denote the valuation of $A$ corresponding to the ideal $(t)$ and written additively. The colength of an ideal is then $v$ of a generator of that ideal. The lemma implies that $D^{m(p-1)+1} t=c^{m} D t$ for $m \geqq 1$. Taking $m=$ $\left(p^{n}-1\right) /(p-1)$, we find that the colength of $\mathfrak{A}\left(D^{p^{n}}\right)$ is given by

Similarly

$$
i(n)=v\left(D^{p^{n}} t\right)=\left(p^{n-1}+\cdots+1\right) v(c)+v(D t) .
$$

$$
i(n-1)=\left(p^{n-2}+\cdots+1\right) v(c)+v(D t) .
$$

Subtracting, we find that $i(n)-i(n-1)=p^{n-1} v(c)$. But $p$ divides $v(c)$ because $c \in K\left[\left[t^{p}\right]\right]$. Hence, $i(n) \equiv i(n-1)$ (modulo $\left.p^{n}\right)$ and the conjecture is true in the one-variable case.

3. Partial result when $A=K\left[\left[t_{1}, \cdots, t_{r}\right]\right]$. The congruence $i(1) \equiv i(0)$ (modulo $p$ ) may be obtained with the aid of the residue symbol. (See Hartshorne [1, pp. 195-199].)

When $\omega$ is an $r$-differential form on $A$ over $K$ and the ideal $\left(f_{1}, \cdots, f_{r}\right)$ has finite colength, the residue symbol

$$
\left[\begin{array}{c}
\omega \\
f_{1}, \cdots, f_{r}
\end{array}\right]
$$

taking values in $K$ is defined. We make use of the following properties, assuming in each case that the conditions for the existence of the residue symbol are fulfilled:

(a) Let $\mathfrak{U}=\left(f_{1}, \cdots, f_{r}\right)$. Then

$$
\left[\begin{array}{c}
d f_{1} \wedge \cdots \wedge d f_{r} \\
f_{1}, \cdots, f_{r}
\end{array}\right]=\text { colength( }(\mathfrak{U}) \cdot 1_{K} .
$$


(b) If $\alpha \in \mathfrak{A}=\left(f_{1}, \cdots, f_{r}\right)$, then

$$
\left[\begin{array}{c}
\alpha \omega \\
f_{1}, \cdots, f_{r}
\end{array}\right]=0 \text {. }
$$

(c) If the elements $g_{i}=\sum_{j=1}^{r} \alpha_{i j} f_{j}$, for $i=1, \cdots, r$, generate another ideal of finite colength, then

$$
\left[\begin{array}{c}
\operatorname{det}\left(\alpha_{i j}\right) \omega \\
g_{1}, \cdots, g_{r}
\end{array}\right]=\left[\begin{array}{c}
\omega \\
f_{1}, \cdots, f_{r}
\end{array}\right] .
$$

Lemma. Suppose that $\left(f_{1}, \cdots, f_{r}\right)$ has finite colength and $\alpha_{i j}, \beta_{i j}$ are elements of $A$ such that

$$
g_{i}=\sum_{j=1}^{r} \alpha_{i j} f_{j}=\sum_{j=1}^{r} \beta_{i j} f_{j}, \quad i=1, \cdots, r .
$$

Then $\operatorname{det}\left(\alpha_{i j}\right)-\operatorname{det}\left(\beta_{i j}\right) \in\left(g_{1}, \cdots, g_{r}\right)$.

Proof. To fix notation, let the Koszul complex $K_{*}\left(f_{1}, \cdots, f_{r}\right)=K_{*}(f)$ be given as follows (see Serre [3, Chapter IV]): $K_{m}(f)=\bigwedge^{m}\left(A^{r}\right)$, the $m$ th exterior product of $A^{r}$. Let $e_{1}, \cdots, e_{r}$ denote the generators of $K_{1}(f)$. The boundary map $\delta_{m}: K_{m}(f) \rightarrow K_{m-1}(f)$ is given by

$$
e_{i_{1}} \wedge \cdots \wedge e_{i_{m}} \rightarrow \sum_{j=1}^{m}(-1)^{j+1} f_{i j} e_{i_{1}} \wedge \cdots \wedge \hat{e}_{i j} \wedge \cdots \wedge e_{i_{m}} .
$$

Let $E_{1}, \cdots, E_{r}$ denote the generators of $K_{1}(g)$. Define a map of $A$ algebras $S_{*}: K_{*}(g) \rightarrow K_{*}(f)$ by the following action on generators: $E_{i} \rightarrow \sum_{j=1}^{r} \alpha_{i j} e_{j}$. Because $g_{i}=\sum_{j=1}^{r} \alpha_{i j} f_{j}$, the following diagram is commutative:

$$
\begin{array}{cc}
K_{1}(g) \stackrel{\delta}{\longrightarrow} & K_{0}(g)=A \\
S_{1} \downarrow & \downarrow^{\text {identity }} \\
K_{1}(f) \stackrel{\delta}{\longrightarrow} & K_{0}(f)=A
\end{array}
$$

and $S$ commutes with the boundary map $\delta$. In particular $S_{r}$ is multiplication by $\operatorname{det}\left(\alpha_{i j}\right)$ in dimension $r$.

Similarly, we define $T: K_{*}(g) \rightarrow K_{*}(f)$ by replacing $\alpha_{i j}$ by $\beta_{i j}$ above.

$K_{*}(g)$ is free. $K_{*}(f)$ is exact in positive dimensions because the ideal $\left(f_{1}, \cdots, f_{r}\right)$ has finite colength $\left[3, \mathrm{p}\right.$. IV-5]. Since $S_{0}=T_{0}=$ identity in dimension zero, there is a standard way (Spanier [5, p. 165]) to construct $h_{m}: K_{m}(g) \rightarrow K_{m+1}(f)$ such that $\delta_{m+1} h_{m}+h_{m-1} \delta_{m}=S_{m}-T_{m}$. In dimension $r$, 
we find that

where $\gamma \in\left(g_{1}, \cdots, g_{r}\right)$.

$$
\begin{aligned}
{\left[\operatorname{det}\left(\alpha_{i j}\right)-\operatorname{det}\left(\beta_{i j}\right)\right] e_{1} \wedge \cdots \wedge e_{r} } & =\left(S_{r}-T_{r}\right) E_{1} \wedge \cdots \wedge E_{r} \\
& =h_{r-1} \delta_{r} E_{1} \wedge \cdots \wedge E_{r} \\
& =\gamma e_{1} \wedge \cdots \wedge e_{r}
\end{aligned}
$$

It follows that $\operatorname{det}\left(\alpha_{i j}\right)-\operatorname{det}\left(\beta_{i j}\right) \in\left(g_{1}, \cdots, g_{r}\right)$ and the lemma is proved.

THEOREM. $i(1) \equiv i(0)$ (modulo $p)$.

Proof. Let $\partial_{i} \alpha$ denote the formal partial derivative of $\alpha$ with respect to $t_{i}$. Thus $D \alpha=\sum_{1}^{r} \partial_{i} \alpha D t_{i}$.

Let $D t_{i}=f_{i}$, and $D^{p} t_{i}=F_{i}$. As $\left(F_{1}, \cdots, F_{r}\right) \subset\left(f_{1}, \cdots, f_{r}\right)$ we may write

$$
F_{i}=\sum_{j=1}^{r} c_{i j} f_{j}, \quad i=1, \cdots, r .
$$

Using properties (a) and (c) of the residue symbol, we find that

$i(0) \cdot 1_{K}=\left[\begin{array}{c}\operatorname{det}\left(c_{i j}\right) d f_{1} \wedge \cdots \wedge d f_{r} \\ F_{1}, \cdots, F_{r}\end{array}\right]=\left[\begin{array}{c}\operatorname{det}\left(c_{i j}\right) \operatorname{det}\left(\partial_{i} f_{j}\right) d t_{1} \wedge \cdots \wedge d t_{r} \\ F_{1}, \cdots, F_{r}\end{array}\right]$ and

$$
i(1) \cdot 1_{K}=\left[\begin{array}{c}
d F_{1} \wedge \cdots \wedge d F_{r} \\
F_{1}, \cdots, F_{r}
\end{array}\right]=\left[\begin{array}{c}
\operatorname{det}\left(\partial_{i} F_{j}\right) d t_{1} \wedge \cdots \wedge d t_{r} \\
F_{1}, \cdots, F_{r}
\end{array}\right] .
$$

Subtracting, we find that

$$
[i(1)-i(0)] \cdot 1_{K}=\left[\begin{array}{c}
\gamma d t_{1} \wedge \cdots \wedge d t_{r} \\
F_{1}, \cdots, F_{r}
\end{array}\right]
$$

where $\gamma=\operatorname{det}\left(\partial_{i} F_{j}\right)-\operatorname{det}\left(c_{i j}\right) \operatorname{det}\left(\partial_{i} f_{j}\right)$.

We now obtain two expressions for $g_{i}=D^{p+1} t_{i}$ by using the fact that both $D$ and $D^{p}$ are derivations.

and

$$
g_{i}=D\left(D^{p} t_{i}\right)=D\left(F_{i}\right)=\sum_{j=1}^{r}\left(\partial_{j} F_{i}\right) f_{j}
$$

$$
g_{i}=D^{p}\left(D t_{i}\right)=D^{p}\left(f_{i}\right)=\sum_{j=1}^{r}\left(\partial_{j} f_{i}\right) F_{j} .
$$

Substitute the expression in (1) for $F_{j}$ above and apply the lemma of this section to show that $\gamma=\operatorname{det}\left(\partial_{i} F_{j}\right)-\operatorname{det}\left(c_{i j}\right) \operatorname{det}\left(\partial_{i} f_{j}\right)$ is an element of the ideal $\left(g_{1}, \cdots, g_{r}\right) \subset\left(F_{1}, \cdots F_{r}\right)$.

Now, by property (b) of the residue symbol, $[i(1)-i(0)] \cdot 1_{K}=0$. Hence the theorem is proved. 


\section{REFERENCES}

1. R. Hartshorne, Residues and duality, Lecture Notes in Math., no. 20, SpringerVerlag, Berlin and New York, 1966. MR 36 \#5145.

2. S. Sen, On automorphisms of local fields, Ann. of Math. (2) 90 (1969), 33-46.

3. J.-P. Serre, Algèbre locale, multiplicités, Cours au Collége de France, 1957-1958, rédigé par Pierre Gabriel, 2 ième éd., Lecture Notes in Math., vol. 11, Springer-Verlag, Berlin and New York, 1965. MR 34 \#1352.

4. - Sur la rationalité des représentations d'Artin, Ann. of Math. (2) 72 (1960), 405-420. MR 30 \#2002.

5. E. H. Spanier, Algebraic topology, McGraw-Hill, New York, 1966. MR 35 \#1007.

Department of Mathematics, Harvard University, Cambridge, Massachusetts 02138

Current address: Department of Mathematics, Queens College, Flushing, New York 11367 